Internat. J. Math. \& Math. Sci.

Vol. 24, No. 11 (2000) 785-791

S0161171200002325

(C) Hindawi Publishing Corp.

\title{
ON SOME TOPOLOGICAL PROPERTIES OF GENERALIZED DIFFERENCE SEQUENCE SPACES
}

\author{
MIKAIL ET
}

(Received 22 October 1998 and in revised form 12 June 2000)

\begin{abstract}
We obtain some topological results of the sequence spaces $\Delta^{m}(X)$, where $\Delta^{m}(X)=\left\{x=\left(x_{k}\right):\left(\Delta^{m} x_{k}\right) \in X\right\},(m \in \mathbb{N})$, and $X$ is any sequence space. We compute the $p \alpha$-, $p \beta$-, and $p \gamma$-duals of $l_{\infty}, c$, and $c_{0}$ and we investigate the $N$-(or null) dual of the sequence spaces $\Delta^{m}\left(l_{\infty}\right), \Delta^{m}(c)$, and $\Delta^{m}\left(c_{0}\right)$. Also we show that any matrix map from $\Delta^{m}\left(l_{\infty}\right)$ into a $B K$-space which does not contain any subspace isomorphic to $\Delta^{m}\left(l_{\infty}\right)$ is compact.
\end{abstract}

Keywords and phrases. Difference sequence spaces, statistical convergence, the $N$-dual, the $p \alpha$-, $p \beta$-, and $p \gamma$-dual.

2000 Mathematics Subject Classification. Primary 40A05, 40C05, 46A45.

1. Introduction. $w$ denotes the space of all scalar sequences and any subspace of $w$ is called a sequence space. The following sequence spaces will be used in what follows:

$l_{\infty}$, the space of all bounded scalar sequences;

$c$, the space of all convergent scalar sequences;

$c_{0}$, the space of all null scalar sequences;

$l_{1}$, the space of all absolutely 1 -summable scalar sequences;

$s$, the space of all real sequences;

$s_{0}$, the space of all statistically convergent sequences of real numbers;

$\Delta^{m}\left(l_{\infty}\right)$, the space of all $\Delta^{m}$-bounded scalar sequences;

$\Delta^{m}(c)$, the space of all $\Delta^{m}$-convergent scalar sequences;

$\Delta^{m}\left(c_{0}\right)$, the space of all $\Delta^{m}$-null scalar sequences;

$\Delta^{m}\left(s_{0}\right)$, the space of all $\Delta^{m}$-statistically convergent sequences of real numbers.

It is known that $l_{\infty}, c$, and $c_{0}$ are $B$-spaces (Banach spaces) with their usual norm $\|x\|_{\infty}=\sup _{k}\left|x_{k}\right|$, where $k \in \mathbb{N}=\{1,2, \ldots\}$. The sequence spaces $l_{\infty}\left(\Delta^{m}\right), c\left(\Delta^{m}\right)$, $c_{0}\left(\Delta^{m}\right)$ have been introduced by Et and Çolak [1]. These sequence spaces are $B K$ spaces (Banach coordinate spaces) with norm

$$
\|x\|_{\Delta}=\sum_{i=1}^{m}\left|x_{i}\right|+\left\|\Delta^{m} x\right\|_{\infty},
$$

where $m \in \mathbb{N}, \Delta^{\circ} x=\left(x_{k}\right), \Delta x=\left(x_{k}-x_{k+1}\right), \Delta^{m} x=\left(\Delta^{m} x_{k}\right)=\left(\Delta^{m-1} x_{k}-\Delta^{m-1} x_{k+1}\right)$, and so

$$
\Delta^{m} x_{k}=\sum_{v=0}^{m}(-1)^{v}\left(\begin{array}{c}
m \\
v
\end{array}\right) x_{k+v} .
$$


For convenience we denote these spaces $\Delta^{m}\left(l_{\infty}\right), \Delta^{m}(c)$, and $\Delta^{m}\left(c_{0}\right)$ and call $\Delta^{m}$. bounded, $\Delta^{m}$-convergent, and $\Delta^{m}$-null sequences, respectively. The operators

$$
\Delta^{(m)}, \quad \sum^{(m)}: w \longrightarrow w
$$

are defined by

$$
\begin{aligned}
\Delta^{(1)} x_{k} & =x_{k}-x_{k-1}, \quad \sum^{(1)} x_{k}=\sum_{j=0}^{k} x_{j}, \quad(k=0,1, \ldots), \\
\Delta^{(m)} & =\Delta^{(1)} \Delta^{(m-1)}, \quad \sum^{(m)}=\sum_{0}^{(1)} \sum^{(m-1)}, \quad(m \geq 2),
\end{aligned}
$$

and

$$
\sum_{0}^{(m)} \Delta^{(m)}=\Delta^{(m)} \sum^{(m)}=\mathrm{id},
$$

the identity on $w$ (see [4]).

For any subset $X$ of $w$ let

$$
\Delta^{m}(X)=\left\{x=\left(x_{k}\right):\left(\Delta^{m} x_{k}\right) \in X\right\} .
$$

Now we define

$$
\Delta^{(m)} x_{k}=\sum_{v=0}^{m}(-1)^{v}\left(\begin{array}{c}
m \\
v
\end{array}\right) x_{k-v} .
$$

It is trivial that $\left(\Delta^{m} x_{k}\right) \in X$ if and only if $\left(\Delta^{(m)} x_{k}\right) \in X$, for $X=l_{\infty}, c$ or $c_{0}$. In [4], Malkowsky and Parashar also showed that the sequence spaces $\Delta^{m}\left(l_{\infty}\right), \Delta^{m}(c)$, and $\Delta^{m}\left(c_{0}\right)$ are also $B K$-spaces with norm

$$
\|x\|_{\Delta 1}=\sup _{k}\left|\Delta^{(m)} x_{k}\right| .
$$

It is trivial that the norms (1.1) and (1.8) are equivalent. Obviously

$$
\begin{aligned}
\Delta^{(m)}: \Delta^{(m)}(X) \rightarrow X, & \Delta^{(m)} x=y & =\left(\Delta^{(m)} x_{k}\right), \\
\sum^{(m)}: X \longrightarrow \Delta^{(m)}(X), & \sum^{(m)} x=y & =\left(\sum^{(m)} x_{k}\right)
\end{aligned}
$$

are isometric isomorphism, for $X=l_{\infty}, c$ or $c_{0}$.

Hence $\Delta^{m}\left(l_{\infty}\right), \Delta^{m}(c)$, and $\Delta^{m}\left(c_{0}\right)$ are isometrically isomorphic to $l_{\infty}, c$, and $c_{0}$, respectively. Thus $l_{1}$ is continuous dual of $\Delta^{m}(c)$ and $\Delta^{m}\left(c_{0}\right)$.

Throughout the paper, we write $\sum_{k}$ for $\sum_{k=1}^{\infty}$ and $\lim _{n}$ for $\lim _{n \rightarrow \infty}$.

Let $A=\left(a_{n k}\right)$ be an infinite matrix of complex numbers. Let $E$ and $F$ be $B K$-spaces. We write $A x=\left(A_{n}(x)\right)$ if $A_{n}(x)=\sum_{k} a_{n k} x_{k}$ converges for each $n \in \mathbb{N}$. If $A x=$ $\left(A_{n}(x)\right) \in E$ for each $x=\left(x_{k}\right) \in F$, then we say that $A$ defines a matrix map from $F$ into $E$ and we denote it by $A: F \rightarrow E$. By $(F, E)$ we mean the class of matrices $A$ such that $A: F \rightarrow E$. We denote the set $\{x \in w: A x$ exists and $A x \in E\}$ by $E_{A}$. Note that $A$ is a matrix map from $F$ into $E$ if and only if $F \subseteq E_{A}$. From now on, $E$ unless specified shall denote a $B K$-space. 
In $B$-space $E$, the following statements are equivalent (see [5]).

(i) $\sum_{n} x_{n}$ is unconditionally convergent.

(ii) $\sum_{n} x_{n}$ is weakly subseries convergent; that is, weak $\lim _{n} \sum_{j=1}^{n} x_{k_{j}}$ exists for each increasing sequence $\left(k_{n}\right)$ of positive integers.

(iii) $\sum_{n} x_{n}$ is subseries convergent; that is, norm $\lim _{n} \sum_{j=1}^{n} x_{k_{j}}$ exists with $\left(k_{n}\right)$ above.

(iv) $\sum_{n} x_{n}$ is bounded multiplier convergent; that is, $\sum_{n} x_{n} t_{n}$ exists for each sequence $t=\left(t_{n}\right)$ of bounded scalars.

2. Some properties of $\Delta^{m}(X)$. In this section, we will give some properties of $\Delta^{m}(X)$.

THEOREM 2.1. Let $X$ be a vector space and let $A \subset X$. If $A$ is a convex set, then $\Delta^{m}(A)$ is a convex set in $\Delta^{m}(X)$,

Proof. Let $x, y \in \Delta^{m}(A)$, then $\Delta^{m} x, \Delta^{m} y \in A$. Since $\Delta^{m}$ is linear, we have

$$
\lambda \Delta^{m} x+(1-\lambda) \Delta^{m} y=\Delta^{m}(\lambda x+(1-\lambda) y), \quad(0 \leq \lambda \leq 1) .
$$

Since $A$ is convex $\left(\lambda \Delta^{m} x+(1-\lambda) \Delta^{m} y\right) \in A$ and so $(\lambda x+(1-\lambda) y) \in \Delta^{m}(A)$, $(0 \leq \lambda \leq 1)$.

LEMMA 2.2. Let $m$ be a positive integer. Then

(i) $\Delta^{m}\left(\bigcup_{n=1}^{\infty} A_{n}\right)=\bigcup_{n=1}^{\infty} \Delta^{m}\left(A_{n}\right)$,

(ii) $\Delta^{m}\left(\bigcap_{n=1}^{\infty} A_{n}\right)=\bigcap_{n=1}^{\infty} \Delta^{m}\left(A_{n}\right)$.

The proof is clear.

LEMMA 2.3. Let $X$ be a Banach space and let $A \subset X$. Then

(i) If $A$ is nowhere dense in $X$, then $\Delta^{m}(A)$ is nowhere dense in $\Delta^{m}(X)$.

(ii) If $A$ is dense in $X$, then $\Delta^{m}(A)$ is dense in $\Delta^{m}(X)$.

(iii) $\Delta^{m}(w)=w$, where $m$ is a positive integer.

PRoof. (i) Suppose that $\overline{\bar{A}}=\varnothing$, but $\frac{\circ}{\Delta^{m}(A)} \neq \varnothing$. Then $\bar{A}$ contains no neighborhood and $B(a) \subset \overline{\Delta^{m}(A)}$, where $B(a)$ is a neighborhood (or open ball) of center $a$ and radius $r$. Hence $a \in B(a) \subset \overline{\Delta^{m}(A)}=\Delta^{m}(\bar{A})$. This implies that $\Delta^{m}(a) \in \bar{A}$. So $B\left(\Delta^{m}(a)\right)_{\circ} \cap A \neq \varnothing$. On the other hand, $B\left(\Delta^{m}(a)\right) \cap A \subset \bar{A}$. This contradicts to $\stackrel{\AA}{A}=\varnothing$. Hence $\overline{\Delta^{m}(A)}=\varnothing$.

(ii) and (iii) are trivial.

THEOREM 2.4. (i) The set $\Delta^{m}\left(s_{0}\right)$ is dense in the space $s$.

(ii) The set $\Delta^{m}\left(s_{0}\right)$ is a set of the first Baire category in the space $s$.

(iii) The set $s-\Delta^{m}\left(s_{0}\right)$ is a set of the second Baire category in the space $s$.

Proof. The proof follows from [6, Theorem 3.1], Lemmas 2.2, and 2.3, we recall that the complement $M^{c}$ of a meager (or of the first category) subset $M$ of a complete metric space $X$ is nonmeager (or of the second category).

THEOREM 2.5. $l_{\infty} \cap \Delta^{m}(c)=l_{\infty} \cap \Delta^{m}\left(c_{0}\right)$. 
Proof. It is trivial that $l_{\infty} \cap \Delta^{m}\left(c_{0}\right) \subset l_{\infty} \cap \Delta^{m}(c)$. Now let $x \in l_{\infty} \cap \Delta^{m}(c)$, then $x \in$ $l_{\infty}$ and $\Delta^{m-1} x_{k^{-}} \Delta^{m-1} x_{k+1} \rightarrow l,(k \rightarrow \infty), \Delta^{m-1} x_{k}-\Delta^{m-1} x_{k+1}=l+\varepsilon_{k}\left(\varepsilon_{k} \rightarrow 0, k \rightarrow \infty\right)$. This implies that

$$
l=n^{-1} \Delta^{m-1} x_{1}-n^{-1} \Delta^{m-1} x_{n+1}+n^{-1} \sum_{k=1}^{n} \varepsilon_{k} .
$$

This yields $l=0$ and $x \in l_{\infty} \cap \Delta^{m}\left(c_{0}\right)$.

3. Dual spaces. In this section, we give the $N$-dual (null dual) of the sequence spaces $\Delta^{m}\left(l_{\infty}\right), \Delta^{m}(c)$, and $\Delta^{m}\left(c_{0}\right)$ and the $p \alpha-, p \beta$-, and $p \gamma$-duals of the sequence spaces of $l_{\infty}, c$, and $c_{0}$.

DEFINITION 3.1. Let $X$ be a sequence space and define

$$
\begin{aligned}
X^{\alpha} & =\left\{a=\left(a_{k}\right): \sum_{k}\left|a_{k} x_{k}\right|<\infty, \forall x \in X\right\}, \\
X^{\beta} & =\left\{a=\left(a_{k}\right): \sum_{k} a_{k} x_{k} \text { is convergent }, \forall x \in X\right\}, \\
X^{\gamma} & =\left\{a=\left(a_{k}\right): \sup _{n}\left|\sum_{k} a_{k} x_{k}\right|<\infty, \forall x \in X\right\}, \\
X^{N} & =\left\{a=\left(a_{k}\right): \lim _{k} a_{k} x_{k}=0, \forall x \in X\right\},
\end{aligned}
$$

then $X^{\alpha}, X^{\beta}, X^{\gamma}$, and $X^{N}$ are called the $\alpha$-, $\beta$-, $\gamma^{-}$, and $N$-(or nul) duals of $X$, respectively. It is known that $X \subset Y$, then $Y^{\eta} \subset X^{\eta}$ for $\eta=\alpha$-, $\beta$-, $\gamma^{-}$, and $N$-, and $c_{0}{ }^{N}=l_{\infty}, l_{\infty}{ }^{N}=$ $c^{N}=c_{0}[2,3]$.

LEMMA 3.2 (see [4]). Let $m$ be a positive integer. Then there exist positive constants $M_{1}$ and $M_{2}$ such that

$$
M_{1} k^{m} \leq\left(\begin{array}{c}
m+k \\
k
\end{array}\right) \leq M_{2} k^{m} \quad \forall k=0,1, \ldots
$$

LEMmA 3.3. Let $x \in \Delta^{m}\left(c_{0}\right)$, then $\left(\begin{array}{c}m+k \\ k\end{array}\right)^{-1}\left|x_{k}\right| \rightarrow 0,(k \rightarrow \infty)$.

Proof. The proof is trivial.

THEOREM 3.4. Let $m$ be a positive integer. Then $\left(\Delta^{m}\left(l_{\infty}\right)\right)^{N}=\left(\Delta^{m}(c)\right)^{N}=U_{1}$ and $\left(\Delta^{m}\left(c_{0}\right)\right)^{N}=U_{2}$, where $U_{1}=\left\{a=\left(a_{n}\right):\left(n^{m} a_{n}\right) \in c_{0}\right\}$ and $U_{2}=\left\{a=\left(a_{n}\right)\right.$ : $\left.\left(\sum_{k=0}^{n}\left(\begin{array}{c}n+m-k-1 \\ m-1\end{array}\right) a_{n}\right) \in l_{\infty}\right\}$.

Proof. The proof of the part $\left(\Delta^{m}\left(l_{\infty}\right)\right)^{N}=\left(\Delta^{m}(c)\right)^{N}=U_{1}$ is easy. We show that $\left(\Delta^{m}\left(c_{0}\right)\right)^{N}=U_{2}$. It is clear that $\sum_{k=0}^{n}\left(\begin{array}{c}n+m-k-1 \\ m-1\end{array}\right)=\left(\begin{array}{c}n+m \\ m\end{array}\right)=\left(\begin{array}{c}n+m \\ n\end{array}\right)$. Let $a \in U_{2}$ and $x \in \Delta^{m}\left(c_{0}\right)$. Then

$$
\lim _{n} a_{n} x_{n}=\lim _{n}\left(\sum_{k=0}^{n}\left(\begin{array}{c}
n+m-k-1 \\
m-1
\end{array}\right)\right) a_{n}\left(\sum_{k=0}^{n}\left(\begin{array}{c}
n+m-k-1 \\
m-1
\end{array}\right)\right)^{-1} x_{n}=0 .
$$

Hence $a \in\left(\Delta^{m}\left(c_{0}\right)\right)^{N}$. 
Now let $a \in\left(\Delta^{m}\left(c_{0}\right)\right)^{N}$. Then $\lim _{n} a_{n} x_{n}=0$ for all $x \in \Delta^{m}\left(c_{0}\right)$. On the other hand, for each $x \in \Delta^{m}\left(c_{0}\right)$ there exists one and only one $y=\left(y_{k}\right) \in c_{0}$ such that

$$
x_{n}=\sum_{k=1}^{n}\left(\begin{array}{c}
n+m-k-1 \\
m-1
\end{array}\right) y_{k}=\sum_{k=0}^{n}\left(\begin{array}{c}
n+m-k-1 \\
m-1
\end{array}\right) y_{k}, \quad y_{0}=0,
$$

by (1.9). Hence

$$
\lim _{n} a_{n} x_{n}=\lim _{n} \sum_{k=0}^{n}\left(\begin{array}{c}
n+m-k-1 \\
m-1
\end{array}\right) a_{n} y_{k}=0 \quad \forall y \in c_{0} .
$$

If we take

$$
a_{n k}= \begin{cases}\left(\begin{array}{c}
n+m-k-1 \\
m-1
\end{array}\right) a_{n}, & 1 \leq k \leq n \\
0, & k>n\end{cases}
$$

then, we get

$$
\lim _{n} \sum_{k=0}^{\infty} a_{n k} y_{k}=\lim _{n} \sum_{k=0}^{n}\left(\begin{array}{c}
n+m-k-1 \\
m-1
\end{array}\right) a_{n} y_{k}=0 \quad \forall y \in c_{0} .
$$

Hence $A \in\left(c_{0}, c_{0}\right)$ and so $\sup _{n} \sum_{k=0}^{\infty}\left|a_{n k}\right|=\sup _{n} \sum_{k=0}^{n}\left(\begin{array}{c}n+m-k-1 \\ m-1\end{array}\right)\left|a_{n}\right|<\infty$. This completes the proof.

Now we give a new kind of duals of sequence sets.

DEFINITION 3.5. Let $X$ be a sequence spaces, $p>0$ and define

$$
\begin{aligned}
& X^{p \alpha}=\left\{a=\left(a_{k}\right): \sum_{k}\left|a_{k} x_{k}\right|^{p}<\infty, \forall x \in X\right\}, \\
& X^{p \beta}=\left\{a=\left(a_{k}\right): \sum_{k}\left(a_{k} x_{k}\right)^{p} \text { is convergent }, \forall x \in X\right\}, \\
& X^{p \gamma}=\left\{a=\left(a_{k}\right): \sup _{n}\left|\sum_{k=0}^{n}\left(a_{k} x_{k}\right)^{p}\right|<\infty, \forall x \in X\right\},
\end{aligned}
$$

then $X^{p \alpha}, X^{p \beta}, X^{p \gamma}$ are called the $p \alpha$-, $p \beta$-, and $p \gamma$-duals of $X$, respectively. It can be shown that $X^{p \alpha} \subset X^{p \beta} \subset X^{p \gamma}$. If we take $p=1$ in this definition, then we obtain the $\alpha$-, $\beta$-, and $\gamma$-duals of $X$.

THEOREM 3.6. Let $X$ stand for $l_{\infty}, c$, and $c_{0}$ and $0<p<\infty$. Then $X^{p \eta}=U$, for $\eta=\alpha, \beta$ or $\gamma$, where $U=\left\{a=\left(a_{k}\right): \sum_{k}\left|a_{k}\right|^{p}<\infty\right\}=l_{p}$.

Proof. We give the proof for the case $X=c_{0}$ and $\eta=\alpha$. If $a \in U$, then

$$
\sum_{k}\left|a_{k} x_{k}\right|^{p} \leq \sup _{k}\left|x_{k}\right|^{p} \sum_{k}\left|a_{k}\right|^{p}<\infty
$$

for each $x \in c_{0}$. Hence $a \in\left(c_{0}\right)^{p \alpha}$. 
Now suppose that $a \in\left(c_{0}\right)^{p \alpha}$ and $a \notin U$. Then there is a strictly increasing sequence $\left(n_{i}\right)$ of positive integers $n_{i}$ such that

$$
\sum_{k=n_{i}+1}^{k=n_{i+1}}\left|a_{k}\right|^{p}>i^{p} .
$$

Define $x \in c_{0}$ by $x_{k}=\operatorname{sgn} a_{k} / i$ for $n_{i}<k \leq n_{i+1}$ and $x_{k}=0$ for $1 \leq k \leq n_{1}$. Then we may write

$$
\begin{aligned}
\sum_{k}\left|a_{k} x_{k}\right|^{p} & =\sum_{k=n_{1}+1}^{k=n_{2}}\left|a_{k} x_{k}\right|^{p}+\cdots+\sum_{k=n_{i}+1}^{k=n_{i+1}}\left|a_{k} x_{k}\right|^{p}+\cdots \\
& =\sum_{k=n_{1}+1}^{k=n_{2}}\left|a_{k}\right|^{p}+\cdots+\frac{1}{i^{p}} \sum_{k=n_{i}+1}^{k=n_{i+1}}\left|a_{k}\right|^{p}+\cdots \\
& >1+1+\cdots=\sum_{k} 1=\infty .
\end{aligned}
$$

This contradicts to $a \in\left(c_{0}\right)^{p \alpha}$. Hence $a \in U$. The proof for the cases $X=l_{\infty}$ or $c$ and $\eta=\beta$ or $\gamma$ is similar.

The proofs of Lemmas 3.7 and 3.8 and Theorem 3.10 are easily obtained by using the same techniques of Mishra [5, Lemmas 1 and 2 and Theorem 1], therefore we give them without proofs.

LEMMA 3.7. Let $A: \Delta^{m}\left(l_{\infty}\right) \rightarrow E$ defines a matrix map. If $A$ is weakly compact, then $\sum_{k} a_{k}$ is unconditionally convergent in $E$.

LEMMA 3.8. If $\sum_{k} a_{k}$ is unconditionally convergent in $E$, then $A: \Delta^{m}\left(l_{\infty}\right) \rightarrow E$ defines a matrix map, and $A(\alpha)=\sum_{k} a_{k} \alpha_{k}$ for every $\alpha=\left(\alpha_{k}\right) \in \Delta^{m}\left(l_{\infty}\right)$.

COROLLARY 3.9. If $\sum_{k} a_{k}$ is unconditionally convergent in $E$, then $\Delta^{m}\left(l_{\infty}\right) \subseteq E_{A}$.

THEOREM 3.10. If $A: \Delta^{m}\left(l_{\infty}\right) \rightarrow E$ is a weakly compact matrix map, then $A$ is compact map.

COROLLARY 3.11. Let $E$ be a $B K$-space such that it contains no subspace isomorphic to $\Delta^{m}\left(l_{\infty}\right)$. If $A: \Delta^{m}\left(l_{\infty}\right) \rightarrow E$ defines a matrix map, then $A$ is compact map.

ACKNOWLEDGEMENT. The author wishes to thank Prof. Dr Rifat Çolak for his valuable comments on the manuscript.

\section{REFERENCES}

[1] M. Et and R. Çolak, On some generalized difference sequence spaces, Soochow J. Math. 21 (1995), no. 4, 377-386. MR 97b:40009. Zbl 841.46006.

[2] D. J. H. Garling, The $\beta$ - and $\gamma$-duality of sequence spaces, Proc. Cambridge Philos. Soc. 63 (1967), 963-981. MR 36\#1965. Zbl 161.10401.

[3] H. Kizmaz, On certain sequence spaces. II, Int. J. Math. Math. Sci. 18 (1995), no. 4, 721-724. MR 96j:46005. Zbl 832.40002.

[4] E. Malkowsky and S. D. Parashar, Matrix transformations in spaces of bounded and convergent difference sequences of order $m$, Analysis 17 (1997), no. 1, 87-97. MR 98f:40009. Zbl 872.40002. 
[5] S. K. Mishra, Matrix maps involving certain sequence spaces, Indian J. Pure Appl. Math. 24 (1993), no. 2, 125-132. MR 94e:46019. Zbl 804.47030.

[6] T. Šalát, On statistically convergent sequences of real numbers, Math. Slovaca 30 (1980), no. 2, 139-150. MR 81k:40002. Zbl 437.40003.

Mikail ET: Department of Mathematics, Firat UniVERSity, 23119-ELAZIG, TURKey

E-mail address: mikai 1 et@hotmai 1.com 


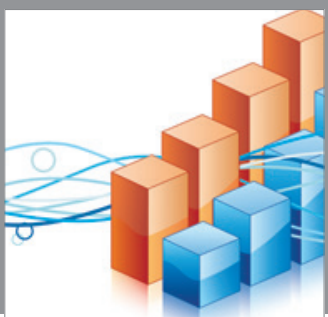

Advances in

Operations Research

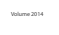

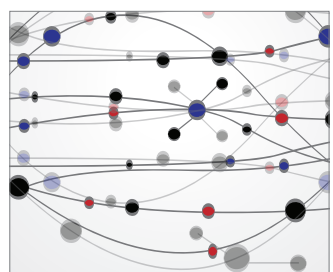

\section{The Scientific} World Journal
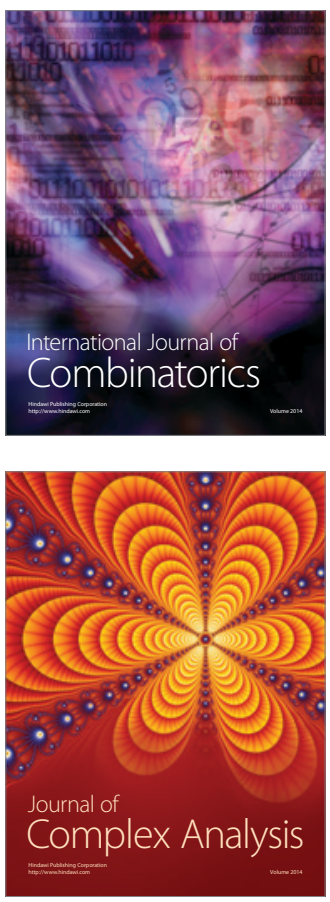

International Journal of

Mathematics and

Mathematical

Sciences
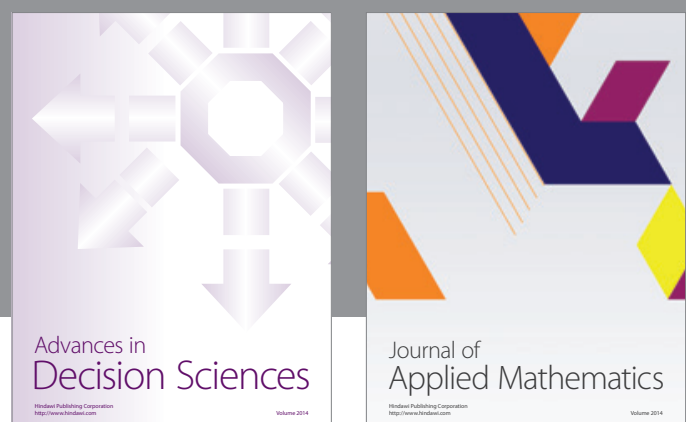

Journal of

Applied Mathematics
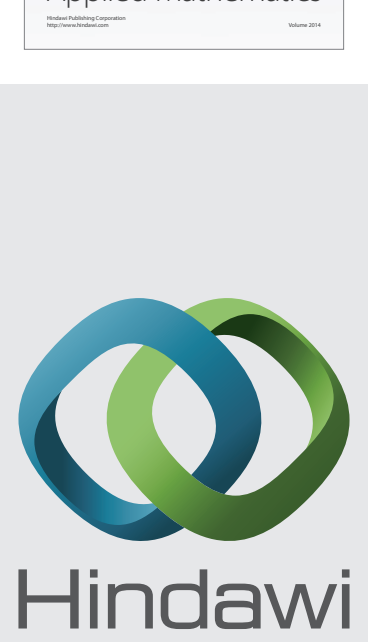

Submit your manuscripts at http://www.hindawi.com
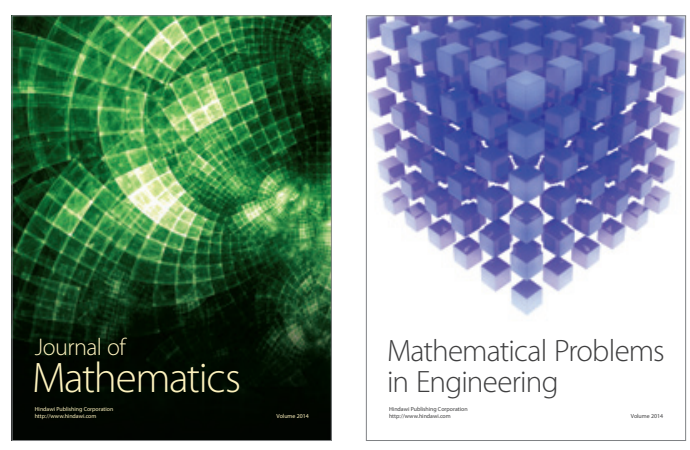

Mathematical Problems in Engineering
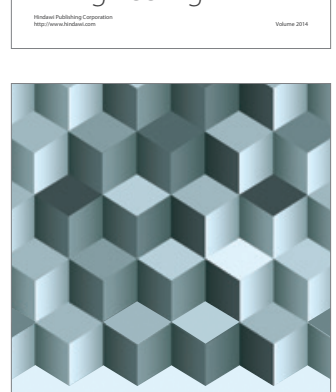

Journal of

Function Spaces
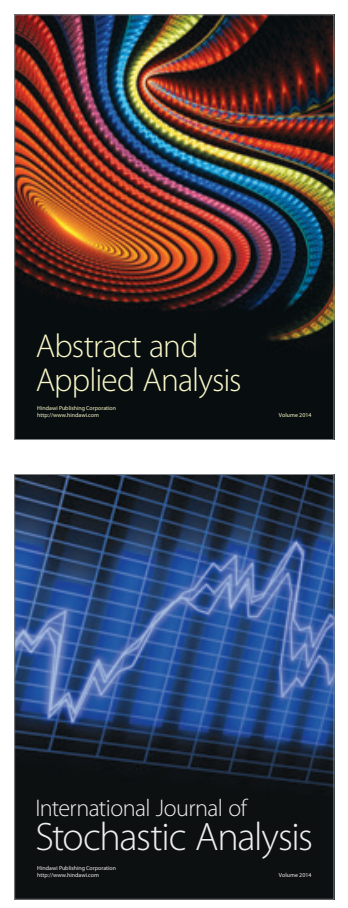

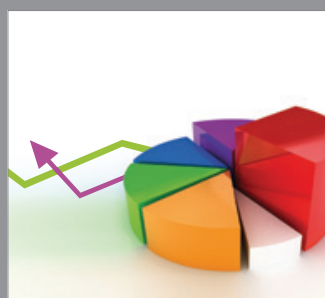

ournal of

Probability and Statistics

Promensencen
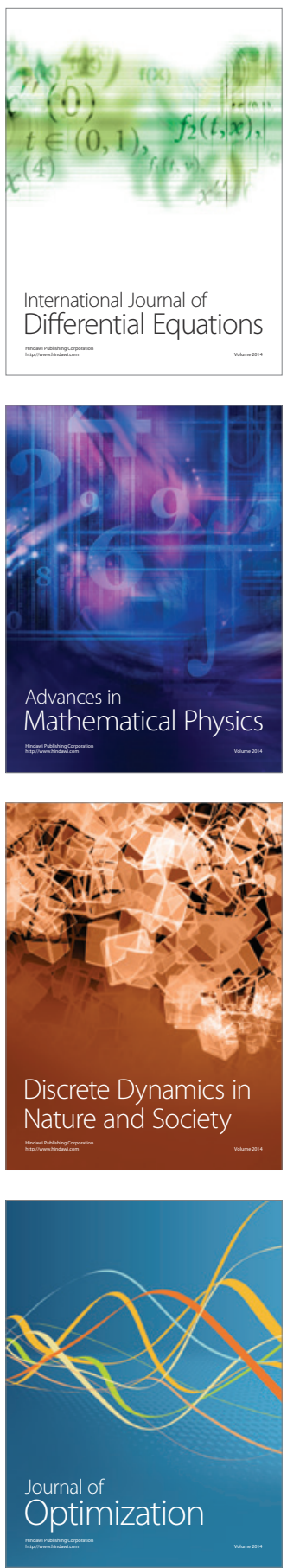